\title{
CCD spectrophotometry of CVs
}

\section{3430-7850 $\AA$ atlas for 27 faint systems}

\author{
U. Munari ${ }^{1}$, T. Zwitter ${ }^{2}$, and A. Bragaglia ${ }^{3}$ \\ 1 Osservatorio Astronomico di Padova, Sede di Asiago, I-36032 Asiago (VI), Italy \\ e-mail: munari@astras.pd.astro.it \\ 2 University of Ljubljana, Department of Physics, Jadranska 19, 1000 Ljubljana, Slovenia \\ e-mail: tomaz.zwitter@uni-lj.si \\ 3 Osservatorio Astronomico di Bologna, via Zamboni 33, Bologna, Italy \\ e-mail angela@astbo3.bo.astro.it
}

Received August 12; accepted August 21, 1996

\begin{abstract}
CCD fluxed spectra are presented for 27 objects from the Downes \& Shara (1993) catalogue of Cataclysmic Variables (CVs). The stars have been selected among those listed as lacking published spectra. As for previous papers in this series, the aim is to check the $\mathrm{CV}$ status of the objects and to provide spectrophotometric data over a wide wavelength range. Integrated $B V R_{\mathrm{C}}$ magnitudes, continuum fluxes at selected wavelengths and integrated fluxes of emission lines are derived for the programme stars. Among the 27 programme stars, 18 show a spectrum supporting the CV classification.
\end{abstract}

Key words: Novae: cataclysmic variables — white dwarfs

\section{Introduction}

Downes \& Shara (1993, hereafter DS93) have compiled a very useful catalogue and atlas of the Cataclysmic Variables discovered through February 1992. A similar work for classical novae was presented by Duerbeck (1987, hereafter D87). DS93 listed references to published quiescence spectra for 271 objects and to outburst spectra for additional 123 systems (in both cases a significant fraction belonging to the pre-digital era), but for 359 objects $(\sim$ half of the total) they have been unable to locate published spectra.

In Papers I, II and III of this series (Zwitter \& Munari 1994-1996) we have begun to present CCD spectra for CVs listed by DS93 as lacking published spectroscopy.

The results of Papers I, II and III confirm the conclusion of DS93 and D87 that the classification of an object

Send offprint requests to: U. Munari as a CV using exclusively the information from photometric observations and/or automated spectral surveys may be misleading. The large fraction of mis-classifications we have encountered so far supports the need for a spectroscopic survey to prune the excellent DS93 catalogue from mis-entries.

In this paper we present flux-calibrated CCD spectra for additional 31 faint systems selected among those without published quiescence spectra according to the DS93 catalogue. The programme continues and observations of additional targets will be presented in future papers of this series.

Beside the confirmation of the CV status, our spectra are intended to expand the set of validated CVs with flux calibrated spectra. A physical analysis of spectral characteristics of the whole investigated sample is postponed to a final paper in this series.

\section{Observations}

The observations listed in Table 1 have been obtained with the $\mathrm{B} \& \mathrm{C}+\mathrm{CCD}$ spectrograph attached to the $1.82 \mathrm{~m}$ telescope operated on Mt. Ekar (Asiago) by the Astronomical Observatory of Padua (Italy). All spectra of programme stars and spectrophotometric standards have been obtained in slitless mode for optimal flux calibration. The observations have been secured during nights with photometric conditions. The covered wavelength range is $3430-7550 \AA$. The spectral resolution is dominated by the dimension of the seeing PSF, on the average $20 \AA$. The Asiago spectra are presented in Figs. 1 to 5.

The objects listed in Table 2 have been observed with the BFOSC+CCD Imager \& Spectrograph attached to the $1.5 \mathrm{~m}$ telescope operated in Loiano by the Astronomical Observatory of Bologna (Italy). The slit spectrograph was 
Table 1. Journal of observations of programme stars observed at Asiago. Type, Max and Min as listed by DS93. Code for spectral information available in literature as given by DS93: $N=$ no spectral information; $D=$ only a glass plate tracing or a description of the quiescence spectrum. Status of the object when we have observed it: $Q=$ quiescence; Out $=$ outburst

\begin{tabular}{|c|c|c|c|c|c|c|c|c|c|c|c|c|}
\hline Name & $\alpha_{2000.0}$ & $\delta_{2000.0}$ & Туре & $\begin{array}{l}\operatorname{Max} \\
(\mathrm{mag})\end{array}$ & & $\begin{array}{r}\text { Min } \\
(\mathrm{mag})\end{array}$ & & Code & $\begin{array}{c}\text { Observ. } \\
\text { date }\end{array}$ & UT & $\begin{array}{l}\text { Expt. } \\
\text { time }\end{array}$ & Status \\
\hline V513 Cas & 001815.05 & +661812.7 & ugZ: & 15.5 & $\mathrm{p}$ & $<17.2$ & $\mathrm{p}$ & $\mathrm{N}$ & 02.11 .95 & 2206 & 30 & Q \\
\hline V410 Cas & 002327.75 & +614627.7 & $\mathrm{NL}^{*}$ & 15.5 & $\mathrm{p}$ & 18 & $\mathrm{p}$ & $\mathrm{N}$ & 02.11 .95 & 2300 & 30 & Q \\
\hline AM Cas & 022623.41 & +711832.2 & ugz & 12.3 & $\mathrm{p}$ & 15.2 & $\mathrm{p}$ & $\mathrm{D}$ & 03.11 .95 & 0007 & 30 & Out \\
\hline KW And & 023518.12 & +411401.6 & UG & 14.8 & $\mathrm{p}$ & 22 & $\mathrm{p}$ & $\mathrm{N}$ & 03.11 .95 & 0106 & 30 & Out \\
\hline PV Per & 024253.57 & +380402.6 & $\mathrm{ug}$ & 14.9 & $\mathrm{p}$ & 20 & $: p$ & $\mathrm{D}$ & 03.11 .95 & 2328 & 30 & $\mathrm{Q}$ \\
\hline MY Per & 040912.33 & +482206.1 & UG & 16 & $\mathrm{p}$ & $<18$ & $\mathrm{p}$ & $\mathrm{N}$ & 04.11 .95 & 0200 & 30 & Q \\
\hline V1062 Tau & $0502 \quad 27.54$ & +244522.1 & $\mathrm{dq}$ & 15.6 & V & & & $\mathrm{N}$ & 04.11 .95 & 0130 & 30 & Q \\
\hline V1504 Cyg & 192857. & +430536. & ugsu & 13.5 & $\mathrm{p}$ & 17.4 & $\mathrm{p}$ & $\mathrm{N}$ & 02.11 .95 & 1918 & 30 & Out \\
\hline $1 \mathrm{H} 1933+510$ & 193436.63 & +510737.3 & $\mathrm{nl}$ & 17.5 & V & & & $\mathrm{N}$ & 03.11 .95 & 1821 & 30 & $\mathrm{Q}$ \\
\hline V811 Cyg & 194823.42 & +362622.7 & ugSS & 12.7 & $\mathrm{p}$ & $<17.7$ & $\mathrm{p}$ & $\mathrm{N}$ & 03.11 .95 & 1856 & 30 & Q \\
\hline V1075 Cyg & 212918.47 & +421916.9 & UGSS & 16.4 & $\mathrm{p}$ & $<18$ & $\mathrm{p}$ & $\mathrm{N}$ & 03.11 .95 & 2000 & 30 & Out? \\
\hline NQ Lac & 222843.94 & +561457.9 & $\mathrm{UG}$ & 16.2 & $\mathrm{p}$ & $<17.7$ & $\mathrm{p}$ & $\mathrm{N}$ & 03.11 .95 & 2106 & 30 & Out? \\
\hline CG Cep & 231026.17 & +663330.6 & ugSS & 14.5 & $\mathrm{p}$ & 17.2 & $\mathrm{p}$ & $\mathrm{N}$ & 03.11 .95 & 2143 & 30 & Out? \\
\hline LM Cas & 231259.71 & +565116.5 & ugSS & 15.8 & $\mathrm{p}$ & $<19$ & $\mathrm{p}$ & $\mathrm{N}$ & 03.11 .95 & 2218 & 30 & Q \\
\hline
\end{tabular}

Table 2. Journal of observations of programme stars observed at Loiano. Type, Max and Min as listed by DS93. Code for spectral information available in literature as given by DS93: $N=$ no spectral information; $D=$ only a glass plate tracing or a description of the quiescence spectrum

\begin{tabular}{|c|c|c|c|c|c|c|c|c|c|c|c|}
\hline Name & $\alpha_{2000.0}$ & $\delta_{2000.0}$ & Type & $\begin{array}{l}\text { Max } \\
\text { (mag) }\end{array}$ & & $\begin{array}{r}\text { Min } \\
(\mathrm{mag})\end{array}$ & & Code & $\begin{array}{c}\text { Observ. } \\
\text { date }\end{array}$ & $\mathrm{UT}$ & $\begin{array}{l}\text { Expt. } \\
\text { time }\end{array}$ \\
\hline$\overline{\mathrm{AM} \text { Cas }}$ & 022623.41 & +711832.2 & ugz & 12.3 & $\mathrm{p}$ & 15.2 & $\mathrm{p}$ & $\mathrm{D}$ & 07.02 .95 & 2059 & 90 \\
\hline V344 Ori & $\begin{array}{llll}06 & 15 & 19.02\end{array}$ & +153058.6 & UGZ & 14.2 & $\mathrm{p}$ & 17.5: & $\mathrm{p}$ & $\mathrm{N}$ & 08.02 .96 & 2001 & 120 \\
\hline SY Gem & 064037. & +311126. & n:: & 9.2 & $\mathrm{v}$ & $<13$ & $\mathrm{v}$ & $\mathrm{N}$ & 14.10 .94 & 0113 & 10 \\
\hline $\mathrm{AQ} \mathrm{CMi}$ & 071434.84 & +084805.0 & UG: & 14.5 & $\mathrm{p}$ & $<16.5$ & $\mathrm{p}$ & $\mathrm{N}$ & 06.02 .95 & 2039 & 45 \\
\hline EG Cnc & 084304.12 & +275148.6 & nl/ug: & 11.9 & $\mathrm{v}$ & 17: & $\mathrm{v}$ & $\mathrm{N}$ & 09.02 .96 & 2003 & 150 \\
\hline $1 \mathrm{H} 0928+500$ & 093215.03 & +495053.4 & $\mathrm{nl}$ & 16.3 & V & & & $\mathrm{N}$ & 15.02 .96 & 2251 & 60 \\
\hline PG $1002+506$ & 100529.40 & +502039.0 & $\mathrm{cV}$ & 15.4 & B & & & $\mathrm{N}$ & 08.02 .96 & 2338 & 60 \\
\hline $1 \mathrm{H} 1025+220$ & 102800.15 & +214812.9 & $\mathrm{nl}$ & 16.2 & V & & & $\mathrm{N}$ & 16.02 .96 & 2213 & 90 \\
\hline PG $1146+228$ & 114900.64 & +223103.6 & $\mathrm{cv}$ & 15.0 & B & & & $\mathrm{N}$ & 07.02 .95 & 2353 & 45 \\
\hline IR Lyr & 182353.62 & +391858.9 & UG: & 14.6 & $\mathrm{p}$ & 16.0 & $\mathrm{p}$ & $\mathrm{N}$ & 03.10 .94 & 2116 & 90 \\
\hline EY Cyg & 195436.85 & +322153.8 & ugSS & 11.4 & $\mathrm{v}$ & 15.5 & $\mathrm{v}$ & $\mathrm{D}$ & 04.07 .94 & 0149 & 35 \\
\hline V476 Cyg & 195824.57 & +533707.1 & na & 2.0 & $\mathrm{p}$ & 17.2 & B & $\mathrm{D}$ & 01.08 .94 & 0056 & 120 \\
\hline HR Del & 204220.18 & +190940.3 & $\mathrm{nb}$ & 3.5 & $\mathrm{v}$ & 12.0 & $\mathrm{v}$ & $\mathrm{D}$ & 06.06 .94 & 0152 & 30 \\
\hline V751 Cyg & 205212.91 & +441925.2 & $\mathrm{nl} / \mathrm{vy}$ & 13.2 & $\mathrm{v}$ & 14.5 & $\mathrm{v}$ & $\mathrm{N}$ & 04.10 .94 & 2129 & 90 \\
\hline
\end{tabular}

set to 2 arcsec, for a resolution of $12 \AA$ and a covered wavelength range between 4000 and $7850 \AA$. In several cases the Loiano observations have been obtained in nonphotometric conditions and thus the zero point of the flux scale is not accurate and no absolute fluxes and $V$ magnitude are derived. The Loiano spectra are presented in Figs. 6 to 10 .

Table 4 gives the classification of the programme stars based on our spectra, Table 5 the integrated flux of selected emission lines, Table 6 the continuum fluxes (at the same reference wavelengths as in Papers I, II and III) and Table 7 the $B V R_{\mathrm{C}}$ magnitudes as derived on our spectra from convolution with the band transmission profiles.

\section{Notes on individual objects}

1H 1025+220, 1H 1933+510. Blue continua with $\mathrm{H} \alpha$, $\mathrm{H} \beta$ and $\mathrm{He} \mathrm{I} 5876 \AA$ in emission.

1H 0928+500. Blue continuum with bright emission lines and He II $4686 \AA$ stronger than $\mathrm{H} \beta$.

PG 1002+506. Our spectrum is remarkably similar to the unpublished one by Ringwald (1993), with $\mathrm{H} \alpha$ in emission but higher Balmer lines in deep, relatively narrow absorption onto of a blue continuum. $\mathrm{H} \beta$ and, to a lesser extent, $\mathrm{H} \gamma$ appear partially filled-in by an emission core. 
Table 3. Target objects without recorded spectra because at the time of our observations they were too faint. Type, Max and Min as listed by DS93. The scheme for spectral code (from DS93) is the same as in Table 2. Last column: magnitude of the object as estimated on the TV screen of the guiding system of the Asiago $1.82 \mathrm{~m}$ telescope

\begin{tabular}{|c|c|c|c|c|c|c|c|c|c|c|}
\hline Name & $\alpha_{2000.0}$ & $\delta_{2000.0}$ & Туре & $\begin{array}{l}\text { Max } \\
(\mathrm{mag})\end{array}$ & & $\begin{array}{c}\text { Min } \\
(\mathrm{mag})\end{array}$ & & Code & $\begin{array}{c}\text { UT observ. } \\
\text { date }\end{array}$ & $\begin{array}{c}V \\
(\mathrm{mag})\end{array}$ \\
\hline PG $0051+169$ & 005340.19 & +170942.8 & $\mathrm{cV}$ & 15.6 & B & & & $\mathrm{N}$ & Nov 03.9931995 & $\geq 17.5$ \\
\hline V550 Cyg & 200504.96 & +322122.8 & UGSS & 14.2 & $\mathrm{p}$ & $<17.0$ & $\mathrm{p}$ & $\mathrm{N}$ & Nov 03.7391995 & $\geq 17.0$ \\
\hline V1310 Cyg & 200933 . & +410012. & ugSS & 15.9 & $\mathrm{p}$ & $<19$ & $\mathrm{p}$ & $\mathrm{N}$ & Nov 03.7421995 & $\geq 17.0$ \\
\hline V767 Cyg & 201650.07 & +531223.6 & UGSS & 15.0 & p & $<17.5$ & & $\mathrm{~N}$ & Nov 03.7441995 & $\geq 17.0$ \\
\hline V1390 Cyg & 202823.46 & +390353.4 & ugSU & 16.0 & $\mathrm{p}$ & $<18.1$ & $\mathrm{p}$ & $\mathrm{N}$ & Nov 03.7051995 & $\geq 16.5$ \\
\hline V632 Cyg & 213604.18 & +402618.8 & UGSS & 12.6 & p & 17.5 & $\mathrm{p}$ & $\mathrm{N}$ & Nov 02.8041995 & $\geq 15.5$ \\
\hline V1089 Cyg & 214418 & +475450 & UGSS & 15.0 & p & 18.5 & $\mathrm{p}$ & $\mathrm{N}$ & Nov 03.8641995 & $\geq 17.0$ \\
\hline MR Lac & 222443.05 & +503140.1 & ug & 15.0 & $\mathrm{p}$ & $<17.3$ & $\mathrm{p}$ & $\mathrm{N}$ & Nov 03.8681995 & $\geq 17.0$ \\
\hline BV And & 232702.19 & +500712.1 & UGSS & 15.2 & $\mathrm{p}$ & 18 & $\mathrm{p}$ & $\mathrm{D}$ & Nov 03.9851995 & $\geq 17.0$ \\
\hline
\end{tabular}

Table 4. Classification from spectra of the programme stars

\begin{tabular}{ll|ll|ll|ll}
\hline Name & & Name & & Name & & Name & \\
\hline 1H 0928+500 & CV & AQ CMi & CV & V476 Cyg & CV & NQ Lac & sdO \\
1 H 1025+220 & CV & AM Cas & CV & V751 Cyg & CV & IR Lyr & not a CV \\
1 H 1933+510 & CV & LM Cas & CV ? & V811 Cyg & CV & V344 Ori & CV \\
PG 1002+506 & CV ? & V410 Cas & $?$ & V1075 Cyg & CV ? & MY Per & $?$ \\
PG 1146+228 & sdO & V513 Cas & $?$ & V1504 Cyg & CV & PV Per & CV \\
KW And & CV & CG Cep & CV & HR Del & CV & V1062 Tau & CV \\
EG Cnc & CV & EY Cyg & CV & SY Gem & $?$ & & \\
\hline
\end{tabular}

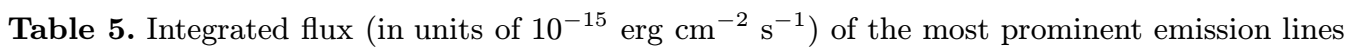

\begin{tabular}{|c|c|c|c|c|c|c|c|c|c|}
\hline & $\mathrm{H} \alpha$ & $\mathrm{H} \beta$ & $\overline{\mathrm{H} \gamma}$ & $\mathrm{H} \delta$ & $\begin{array}{c}\text { He I } \\
(4471 \AA)\end{array}$ & $\begin{array}{c}\text { He I } \\
(5876 \AA)\end{array}$ & $\begin{array}{c}\text { He I } \\
(6678 \AA)\end{array}$ & $\begin{array}{c}\text { He I } \\
(7065 \AA)\end{array}$ & $\begin{array}{c}\text { He II } \\
(4686 \AA)\end{array}$ \\
\hline V513 Cas & 6 & & & & & & & & \\
\hline AM Cas (Loiano) & 25 & 20 & 15 & & & & & & \\
\hline MY Per & 3 & & & & & & & & \\
\hline V1062 Tau & 24 & 10 & & & & & & & \\
\hline V811 Cyg & 50 & 52 & 46 & 43 & 20 & 17 & 7 & 5 & 10 \\
\hline CG Cep & 12 & & & & & & & & \\
\hline V751 Cyg & 18 & 7 & 8 & & & & & & \\
\hline PG $1002+506$ & 20 & & & & & & & & \\
\hline EY Cyg & 9 & 6 & & & & & & & \\
\hline $1 \mathrm{H} 1025+220$ & 10 & 7 & & & & & & & \\
\hline $1 \mathrm{H} 0928+500$ & 6 & 6 & 7 & & & & & & 10 \\
\hline V476 Cyg & 5 & 6 & & & & & & & 3 \\
\hline AQ Cmi & 3 & & & & & & & & \\
\hline
\end{tabular}

Ringwald found a fine sinusoidal modulation of the $\mathrm{H} \alpha$ radial velocity with an amplitude of $30 \mathrm{~km} \mathrm{~s}^{-1}$ and a period of 7.6 hours. However he dismissed a CV classification for PG $1002+506$ and ascribed the RV modulation to some sort of noise and/or uneven sampling of the data.

The colors of our spectrum suggest little or absent reddening, in agreement with the very high galactic latitude $\left(b=+51^{\circ}\right)$. With an observed $V=15.5 \mathrm{mag}$ the object would lie at $d \sim 10 \mathrm{kpc}$ if an analogue of an Herbig
$A \mathrm{e} / B \mathrm{e}$ star, with an implausible large $z \sim 8 \mathrm{kpc}$ for a young object. Alternatively, it could be a sdB-O star in a close binary or more probably a CV seen nearly pole-on (in agreement with the sharpness of absorption lines). The object deserves further investigation.

PG 1146+228. Again our spectrum is identical with the unpublished one by Ringwald (1993), with a blue continuum and absorption lines by hydrogen and possibly He II 
Table 6. Continuum fluxes for the programme stars. The fluxes have been scaled to Flux $(5200 \AA)=1.00$. They were computed averaging over $50 \AA$ wide bins centered at the given wavelength

\begin{tabular}{|c|c|c|c|c|c|c|c|c|c|}
\hline \multirow[t]{2}{*}{ Name } & \multicolumn{9}{|c|}{ Continuum Fluxes } \\
\hline & 3450 & 3750 & 4000 & 4400 & 4800 & 5200 & 5600 & 6000 & 7050 \\
\hline \multicolumn{10}{|c|}{ Asiago spectra } \\
\hline V513 Cas & & 0.58 & 0.75 & 0.85 & 0.91 & 1.00 & 1.09 & 1.06 & 1.10 \\
\hline V410 Cas & 0.18 & 0.49 & 1.09 & 1.14 & 1.05 & 1.00 & 0.91 & 0.81 & 0.60 \\
\hline AM Cas & & 1.34 & 1.63 & 1.48 & 1.26 & 1.00 & 0.85 & 0.70 & 0.47 \\
\hline PV Per & & & 1.56 & 1.40 & 1.23 & 1.00 & 1.11 & 1.09 & 0.87 \\
\hline V1062 Tau & 1.18 & 1.16 & 1.03 & 0.87 & 0.90 & 1.00 & 1.09 & 1.12 & 1.09 \\
\hline V1504 Cyg & 1.44 & 2.18 & 1.97 & 1.67 & 1.33 & 1.00 & 0.80 & 0.64 & 0.40 \\
\hline $1 \mathrm{H} 1933+510$ & & 2.06 & 1.57 & 1.63 & 1.14 & 1.00 & 0.82 & 0.74 & 0.46 \\
\hline V811 Cyg & & 3.21 & 2.05 & 1.26 & 1.06 & 1.00 & 0.90 & 0.82 & 0.86 \\
\hline V1075 Cyg & & 1.44 & 1.38 & 1.27 & 1.25 & 1.00 & 0.90 & 0.79 & 0.55 \\
\hline NQ Lac & 0.66 & 0.88 & 0.90 & 0.86 & 1.05 & 1.00 & 1.19 & 1.32 & 1.64 \\
\hline CG Cep & 0.63 & 1.22 & 1.22 & 1.15 & 1.09 & 1.00 & 0.91 & 0.86 & 0.70 \\
\hline LM Cas & & 0.61 & 0.72 & 0.99 & 1.06 & 1.00 & 1.05 & 1.03 & 0.91 \\
\hline \multicolumn{10}{|c|}{ Loiano spectra } \\
\hline AM Cas & & & & 1.27 & 1.11 & 1.00 & 0.91 & 0.79 & 0.60 \\
\hline SY Gem \#1 & & & & 0.69 & 1.01 & 1.00 & 1.09 & 1.09 & 0.97 \\
\hline SY Gem \#2 & & & & 1.23 & 1.16 & 1.00 & 0.92 & 0.81 & 0.61 \\
\hline EG Cnc & & & & 1.64 & 1.25 & 1.00 & 0.67 & 0.44 & 0.37 \\
\hline $1 \mathrm{H} 0928+500$ & & & & 1.47 & 1.18 & 1.00 & 0.88 & 0.76 & 0.50 \\
\hline PG $1002+506$ & & & & 1.96 & 1.35 & 1.00 & 0.78 & 0.61 & 0.37 \\
\hline $1 \mathrm{H} 1025+220$ & & & & 1.40 & 1.18 & 1.00 & 0.82 & 0.68 & 0.41 \\
\hline PG $1146+228$ & & & & 1.46 & 1.22 & 1.00 & 0.85 & 0.72 & 0.49 \\
\hline IR Lyr & & & & 1.23 & 1.16 & 1.00 & 0.89 & 0.75 & 0.49 \\
\hline EY Cyg & & & & 0.72 & 1.10 & 1.00 & 1.09 & 1.09 & 1.03 \\
\hline V476 Cyg & & & & 1.42 & 1.34 & 1.00 & 0.92 & 0.83 & 0.66 \\
\hline HR Del & & & & 1.60 & 1.19 & 1.00 & 0.81 & 0.68 & 0.48 \\
\hline V751 Cyg & & & & 1.21 & 1.05 & 1.00 & 0.91 & 0.78 & 0.59 \\
\hline
\end{tabular}

$4686 \AA$. With a galactic latitude $b=+75^{\circ}$ and $V=15.5$ the object must be a sdO.

KW And. Blue continuum with possible weak $\mathrm{H} \alpha$ emission. Comparing with the magnitude range quoted by DS93, our observation at $V=18.7$ suggests the object was in between quiescence and outburst states.

AQ Cmi, V344 Ori, EG Cnc. CV-like spectra, with possibly He II in emission for EG Cnc.

AM Cas. The Asiago spectrum shows an early-type continuum with broad hydrogen lines in deep absorption and only a weak emission core in $\mathrm{H} \alpha$. This corresponds to an outburst state, as confirmed by the bright $V=13.6$ magnitude (cf. Table 7) compared with the range of variability of the star listed by DS93. Adopting the intrinsic colors of a B8 V star, the $B-V$ in Table 7 suggests $E_{B-V}=0.17$ and the $V-R_{\mathrm{C}}$ gives $E_{B-V}=0.16$.
The Loiano spectrum $(V=14.6)$ was secured when the star was far from an outburst maximum but not yet at flat quiescence conditions. Hydrogen emission lines are stronger and He I could be in weak emission. Broad and shallow absorptions with narrow emission cores dominate at higher Balmer lines. The continuum slope is redder compared with the Asiago outburst observation but still bluer than in the spectrum presented by Downes et al. (1995) which was secured during a flat quiescence phase. In the latter spectrum the broad and shallow absorptions in the higher Balmer lines are absent and the He I lines are in much stronger emission.

LM Cas. Our spectrum has been secured when the object was at $B=16.8$, against a range from 15.8 to fainter than 19 according to DS93. The spectrum is featureless, except for a wide absorption at $\mathrm{H} \alpha$. The star could have been caught during an outburst. 
Table 7. $B-V, V-R_{\mathrm{C}}$ colour indices and $V$ magnitude of the programme stars as derived from our spectrophotometry

\begin{tabular}{|c|c|c|c|c|c|c|c|}
\hline Name & $V$ & $B-V$ & $V-R_{\mathrm{C}}$ & Name & $V$ & $B-V$ & $V-R_{\mathrm{C}}$ \\
\hline \multicolumn{4}{|c|}{ Asiago spectra } & \multicolumn{4}{|c|}{ Loiano spectra } \\
\hline V513 Cas & 16.3 & 0.85 & 0.50 & AM Cas & 14.6 & 0.44 & 0.22 \\
\hline V410 Cas & 15.3 & 0.43 & 0.19 & V344 Ori & & 0.37 & 0.54 \\
\hline AM Cas & 13.6 & 0.09 & 0.06 & SY Gem \#1 & 15.1 & 1.08 & 0.45 \\
\hline KW And & 18.7 & 0.42 & 0.39 & SY Gem \#2 & 11.8 & 0.47 & 0.22 \\
\hline PV Per & 19.1 & 0.20 & 0.10 & $\mathrm{AQ} \mathrm{CMi}$ & 18.8 & 0.59 & 0.15 \\
\hline MY Per & 19.8 & 1.51 & 0.67 & EG Cnc & & 0.17 & -0.05 \\
\hline V1062 Tau & 16.6 & 0.70 & 0.46 & $1 \mathrm{H} 0928+500$ & 17.1: & 0.42 & 0.16 \\
\hline V1504 Cyg & 15.2 & -0.08 & 0.00 & PG $1002+506$ & 15.5 & 0.02 & 0.00 \\
\hline $1 \mathrm{H} 1933+510$ & 18.0 & 0.16 & 0.16 & $1 \mathrm{H} 1025+220$ & 16.8: & 0.29 & 0.06 \\
\hline V811 Cyg & 16.8 & 0.10 & 0.32 & PG $1146+228$ & & 0.23 & 0.13 \\
\hline V1075 Cyg & 16.2 & 0.22 & 0.17 & IR Lyr & 14.4 & 0.30 & 0.13 \\
\hline NQ Lac & 15.7 & 0.78 & 0.62 & EY Cyg & 15.8 & 0.77 & 0.45 \\
\hline CG Cep & 15.2 & 0.38 & 0.26 & V476 Cyg & 17.6 & 0.25 & 0.20 \\
\hline \multirow[t]{2}{*}{ LM Cas } & 16.1 & 0.73 & 0.36 & HR Del & & 0.14 & 0.11 \\
\hline & & & & V751 Cyg & 14.3 & 0.36 & 0.20 \\
\hline
\end{tabular}

V410 Cas. Antipin \& Shugarov (1992) described the very unusual lightcurve of this object, characterized by long quiescence (at $B \sim 17 \div 18 \mathrm{mag}$ ) and two major outbursts in 1938 and 1975 , when the star rose to $B=15.5$ Since 1975, the star is at flat maximum. They pointed out photometric similarity with symbiotic novae like PU Vul or HM Sge. Our spectrum is that of a WD of type DA (or possibly that of a CV in outburst, cf. the AM Cas spectrum in the same Fig. 1), with no evidence for emission cores in the broad Balmer absorptions. Object classification is quite obscure given the photometric history. The spectrum has no relation to those of symbiotic stars and symbiotic novae both in quiescence and outbursts, and maximum brightness lasting 20 years makes association with typical CVs very difficult. Enigmatic object deserving further investigations.

V513 Cas. Weak $\mathrm{H} \alpha$ emission on a reddish, featureless continuum. Magnitudes and colors in Table 7 are very close to the values measured by Misselt (1996). Classification uncertain.

CG Cep. At the time of our observation the star was at $B=15.5$ mag against the variability range reported by DS93 as $14.5-17.2$ in blue light. $\mathrm{H} \alpha$ is in moderate emission, $\mathrm{H} \beta$ filled-in and $\mathrm{H} \gamma$ in absorption. The spectrum resembles outburst conditions. Magnitudes and colors index in Table 7 well match those measured by Misselt (1996).

EY Cyg. A clear CV spectrum with He I in emission (He I $5876 \AA$ affected by a cosmic ray), in full agreement with the photographic reproduction of a plate quiescence spectrum by Kraft (1962). A spectrum during decline from maximum has been presented by Szkody et al. (1990), with emission cores onto broad and shallow Balmer lines as above described for AM Cas outburst spectra.

V476 Cyg (= Nova Cyg 1920). A nice CV spectrum for this old nova, with a blue continuum and He II in emission 75 years after the outburst. Magnitude and colors in good agreement with Bruch \& Engel (1994).

V751 Cyg. Our quiescence spectrum was secured at a similar flux level as recorded by Downes et al. (1995). Their spectrum shows only H-alpha in emission on a blue continuum, with two absorption features they identify with the $G$-band and NaI D doublet. Our spectrum suggests that the latter is instead due to He I $5876 \AA$ and the $G$-band actually is part of the broad and shallow Balmer absorption around the $\mathrm{H} \gamma$ core in emission. Magnitude and colors from our spectrum are in good agreement with the value reported by Bruch \& Engels (1994).

V811 Cyg. Text-book example of a CV spectrum (cf. with Fig. 1 in Paper II), with He II in emission.

V1075 Cyg. Our spectrum shows a blue continuum with no perceptible feature at $\mathrm{H} \alpha$. We observed the object at $B=16.4 \mathrm{mag}$, at the maximum of the brightness range listed by DS93. It could be a CV caught during an outburst.

V1504 Cyg. Blue continuum without Balmer jump, weak $\mathrm{H} \alpha$ in emission, possible $\mathrm{H} \gamma$ core in emission inside a broad absorption, higher Balmer lines in absorption. Colors in Table 7 suggest a $E_{B-V}=0.05$. 
HR Del (= Nova Del 1967). Quite surprisingly, such a famous object has no published digital optical spectrum, according to DS93 and the SIMBAD database. The most recent published spectra seems to be the tracings of Kürster \& Barwig (1988), obtained on photographic plates or with electronographic Lallemand cameras in the late 70ies. Our spectrum still show broad [OIII] lines but of now much reduced intensity, strong Balmer lines, He II 4686 and the $4640 \AA$ complex (CIII, NIII) in emission. The He II $(4686 \AA) / \mathrm{H} \beta$ ratio is still close to unity.

SY Gem (= Nova Gem 1856b). Both candidates (stars \#1 and \#2) from the D87 finding chart have been observed. None of them show a CV-like spectrum, in agreement with the photometric data of Downes \& Szkody (1989) and their spectrum description. SY Gem was reported at $V \sim 9.3$ in 1857, 1858, 1904 and 1906, otherwise fainter than mag 10 or 12 , a behaviour quite unusual for real novae. It could be a mis-identified object on the D87 finding chart, or a dwarf nova with erratic outbursts (and quiescence spectra dominated by the cool star) or a different type of variable. Downes \& Skody reported that a nearby star, of $V=20.4 \mathrm{mag}$, could have the $\mathrm{H} \alpha$ in emission.

NQ Lac. We observed the star at $B=16.5$, close to the maximum brightness range reported by DS93. The spectrum is relatively featureless, with Balmer lines in weak absorption together with He II $4686 \AA$. The absence of a strong interstellar NaI D absorption (for a galactic latitude $b=-1$ ) suggests the object to be a sdO.

IR Lyr. Our spectrum shows an A-type absorption continuum, with no trace of emission lines. It is very similar to the one presented by Downes et al. (1995). The object is not a CV.
MY Per. Weak $\mathrm{H} \alpha$ emission on a quite red continuum without molecular bands. Classification uncertain.

PV Per. Blue continuum with weak $\mathrm{H} \alpha$ emission. Dwarf nova outbursts described by Romano \& Minello (1976).

V1062 Tau (=1H 0459+248). H $\alpha, \mathrm{H} \beta$, He I $6678 \AA$ and possibly Balmer continuum appear in emission onto of a reddish continuum. Major differences with the spectrum presented by Remillar et al. (1994) are He II $4686 \AA$ very weak or absent in our spectrum, marked NaI D absorption and absence of He I $5876 \AA$.

Acknowledgements. We would like to thank R. Passuello to have assisted us in securing some of the Asiago spectra.

\section{References}

Antipin S.V., Shugarov S.Yu., 1992, IBVS 3732

Bruch A., Engel A., 1994, A\&AS 104, 79

Downes R.A., Shara M.M., 1993, PASP 105, 127 (DS93)

Downes R.A., Skzoky P., 1989, AJ 97, 1729

Downes R.A., Hoard D.W., Szkody P., Wachter S., 1995, AJ 110,1824

Duerbeck H.W., 1987, A Reference Catalogue and Atlas of Galactic Novae. Reidel (D87)

Kato T., 1995, IBVS 4152

Kraft R.P., 1962, ApJ 135, 408

Kürster M., Barwig H., 1988, A\&A 199, 201

Misselt K.A., 1996, PASP 108, 146

Remillard R.A., et al., 1994, ApJ 428, 785

Ringwald F.A., 1993, Ph.D. Thesis, Dartmouth College, UK

Romano G., Minello S., 1976, IBVS 1140

Szkody P., Piche F., Feinswog L., 1990, ApJS 73, 441

Zwitter T., Munari U., 1994, A\&AS 107, 503 (Paper I)

Zwitter T., Munari U., 1995, A\&AS 114, 575 (Paper II)

Zwitter T., Munari U., 1996, A\&AS 117, 449 (Paper III) 


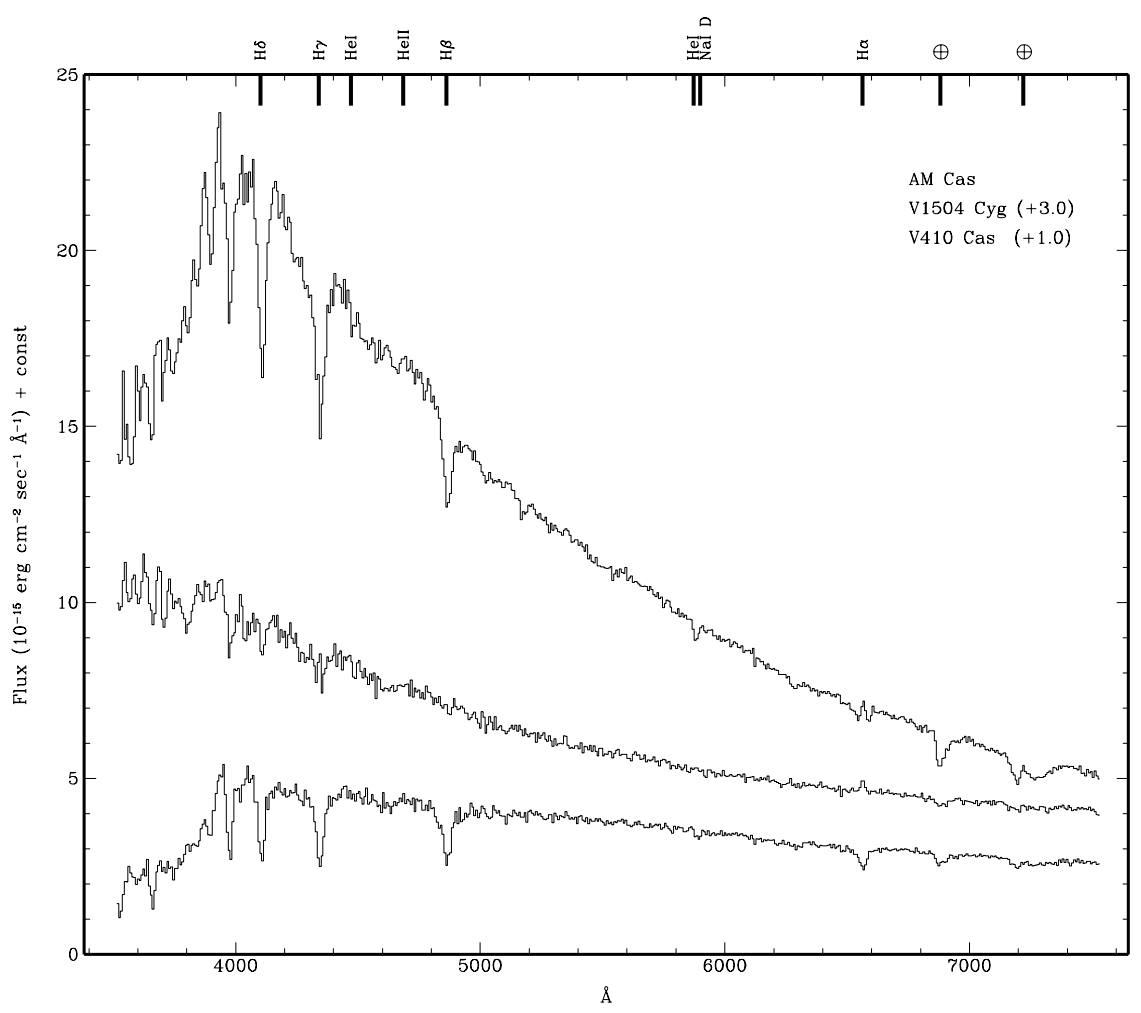

Fig. 1. Spectra of AM Cas (Asiago), V1504 Cyg and V410 Cas. The offset applied for plot clarity is given in brackets next to the star name. The spectra are not corrected for reddening. Fluxes in units of $10^{-15} \mathrm{erg} \mathrm{cm}^{-2} \mathrm{~s}^{-1} \AA^{-1}$

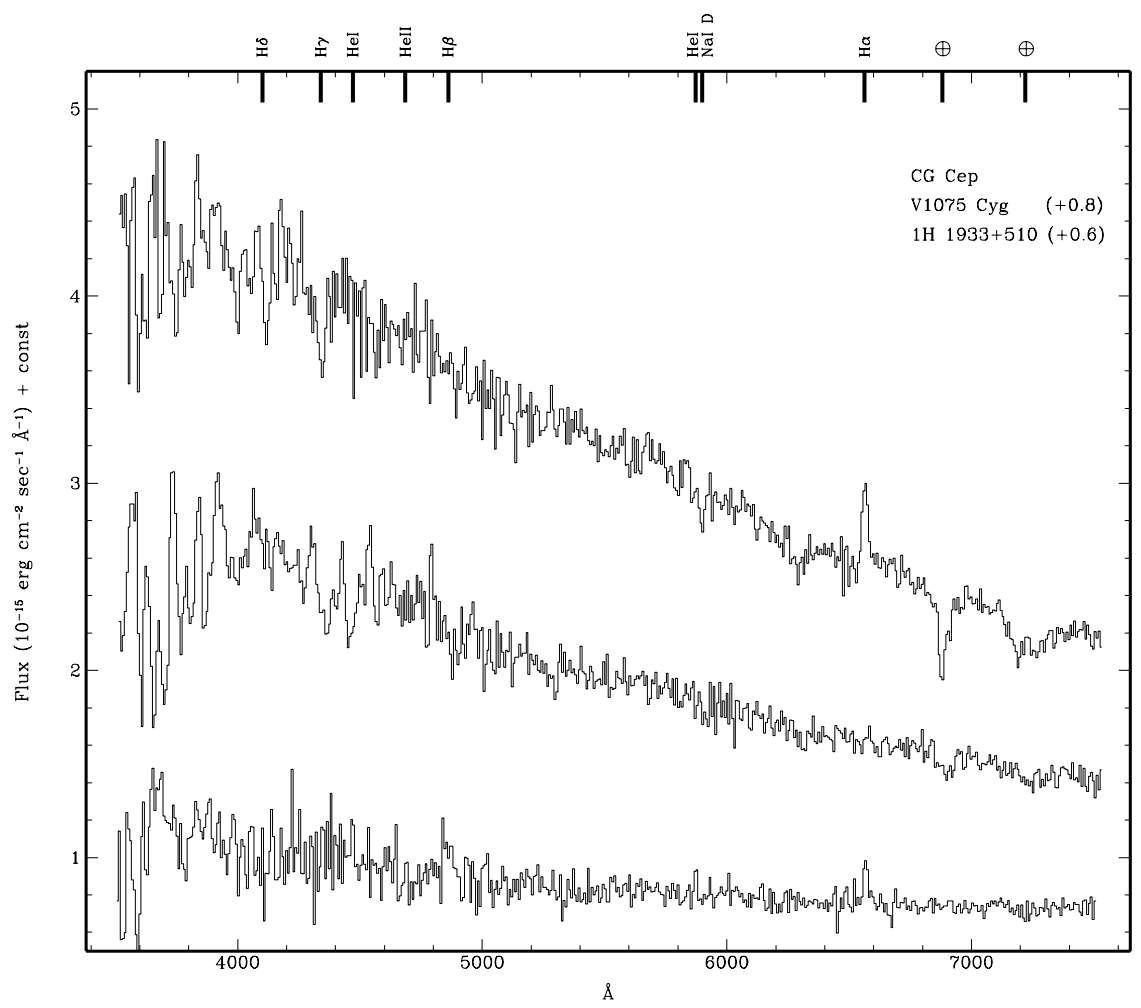

Fig. 2. Spectra of CG Cep, V1075 Cyg and $1 \mathrm{H} 1933+510$. The offset applied for plot clarity is given in brackets next to the star name. The spectra are not corrected for reddening. Fluxes in units of $10^{-15} \mathrm{erg} \mathrm{cm}^{-2} \mathrm{~s}^{-1} \AA^{-1}$ 


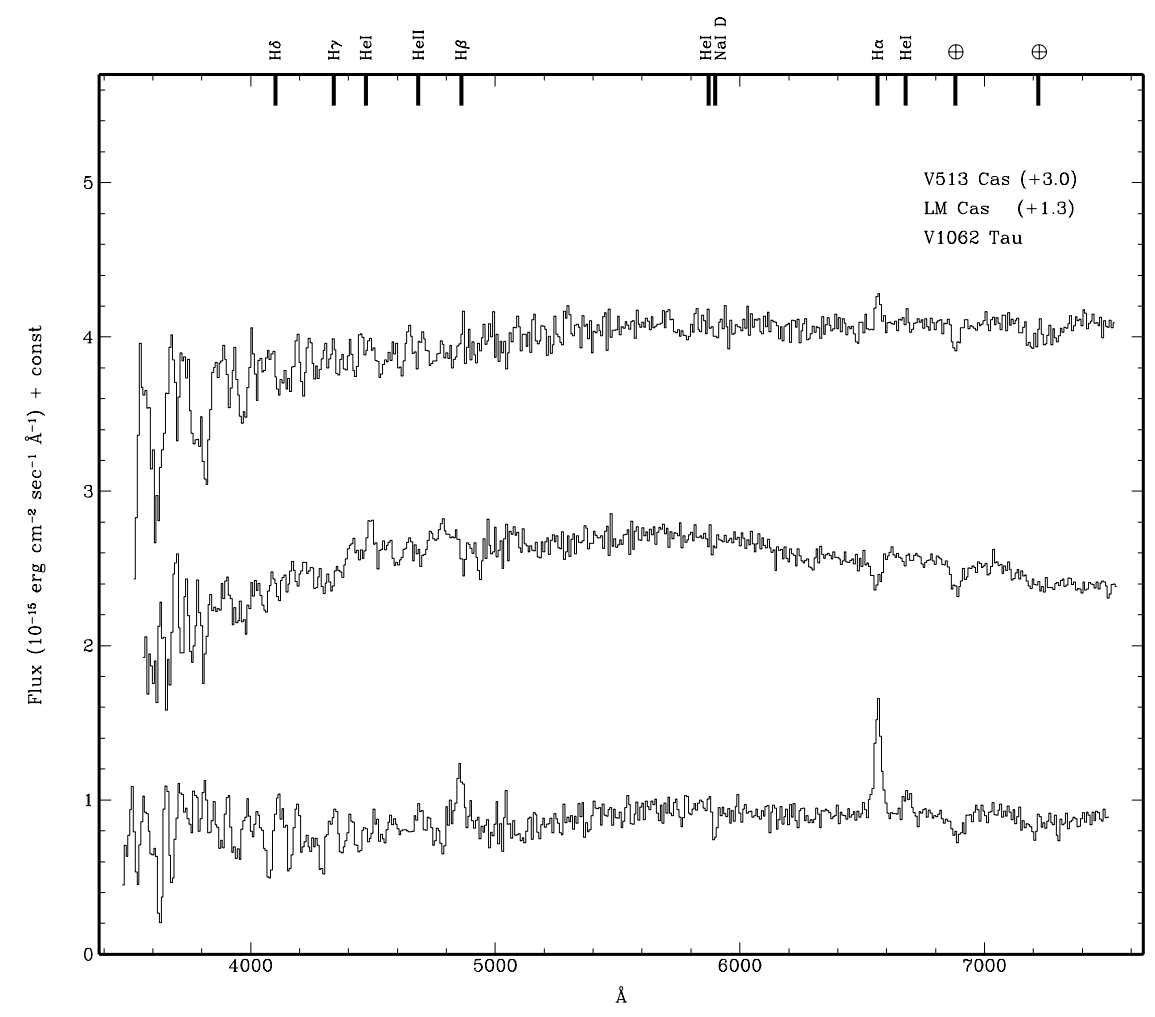

Fig. 3. Spectra of V513 Cas, LM Cas and V1062 Tau. The offset applied for plot clarity is given in brackets next to the star name. The spectra are not corrected for reddening. Fluxes in units of $10^{-15} \mathrm{erg} \mathrm{cm} \mathrm{cm}^{-2} \mathrm{~s}^{-1}$

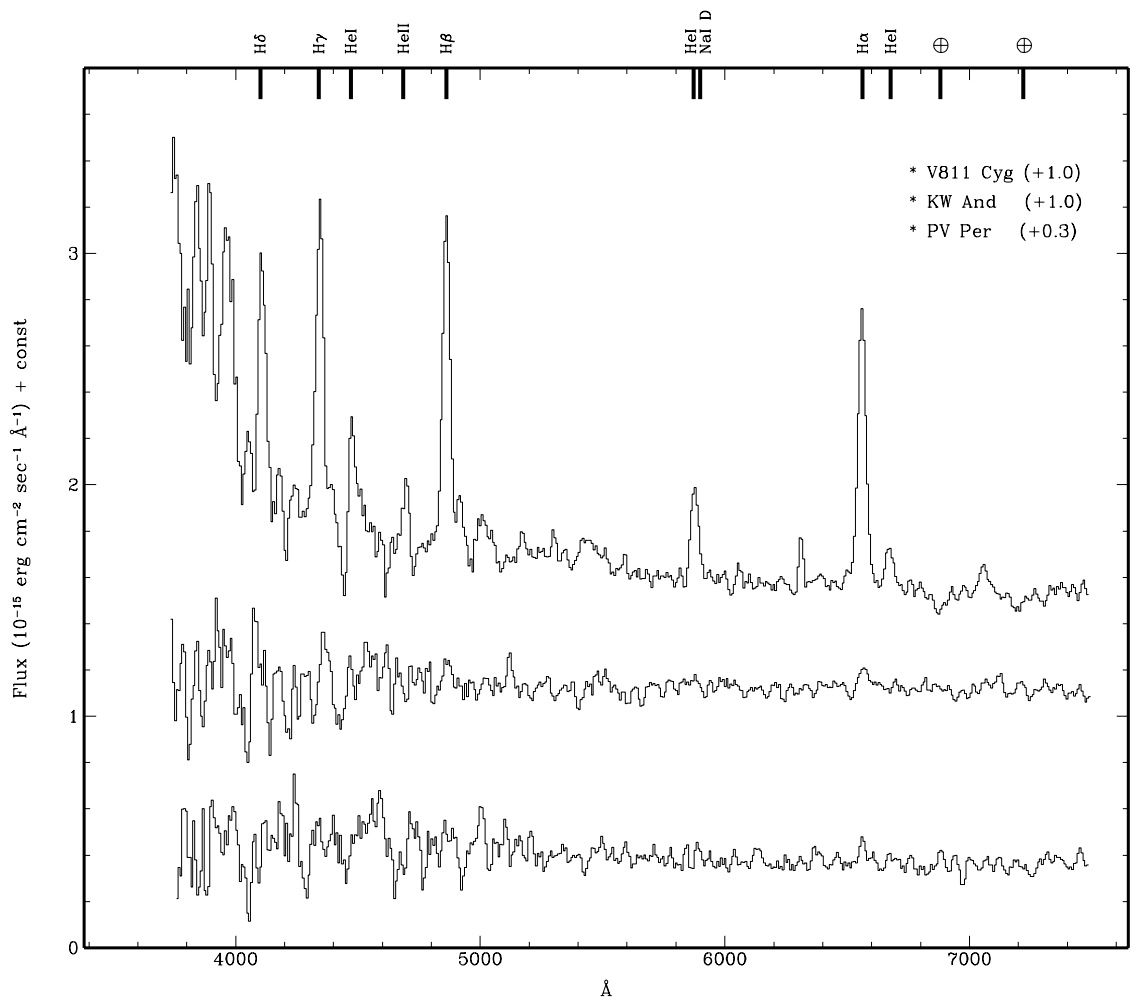

Fig. 4. Spectra of V811 Cyg, KW And and PV Per. The offset applied for plot clarity is given in brackets next to the star name. The asterisk means that a boxcar smoothing (with a window of 3 pixels) has been applied. The spectra are not corrected for reddening. Fluxes in units of $10^{-15} \mathrm{erg} \mathrm{cm}^{-2} \mathrm{~s}^{-1} \AA^{-1}$ 


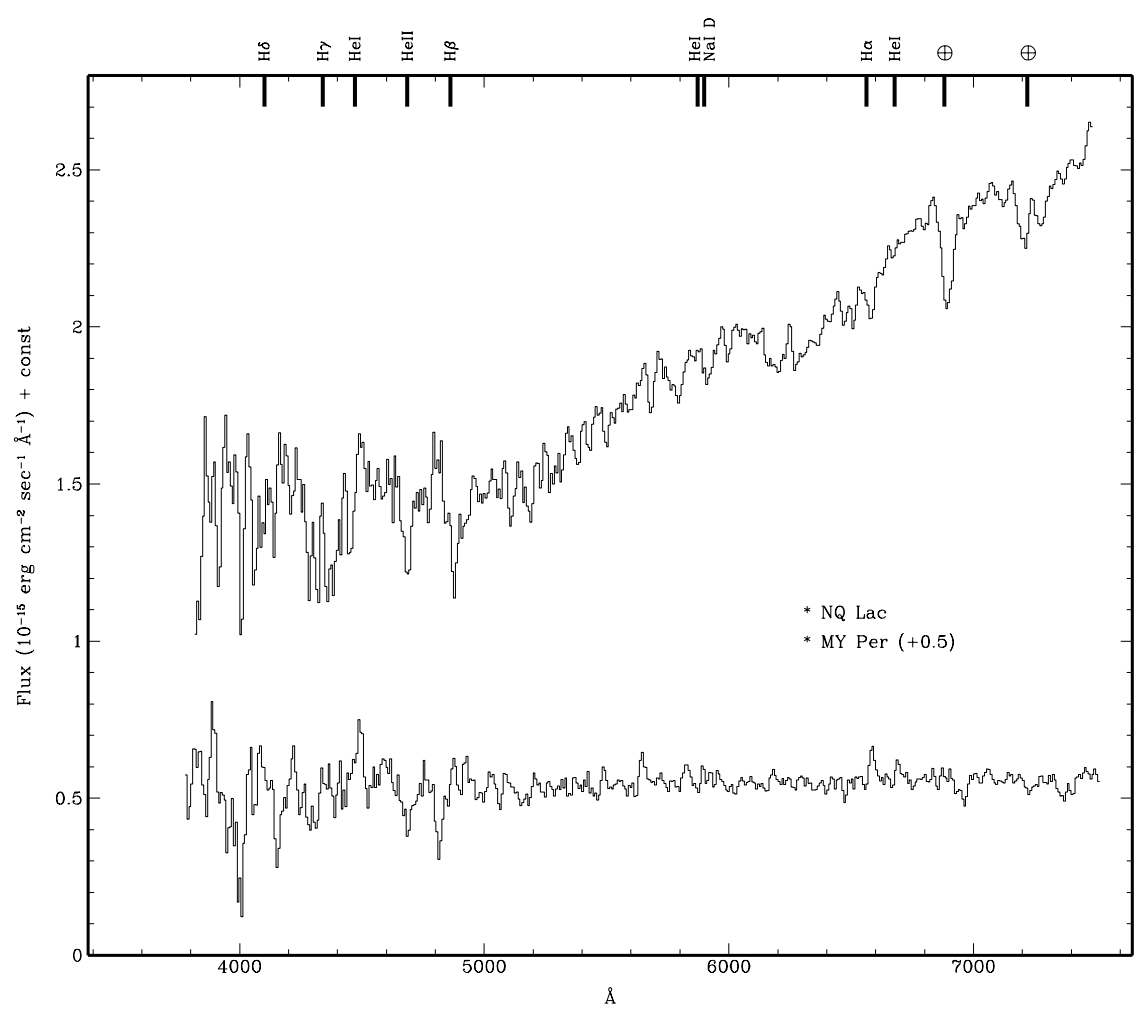

Fig. 5. Spectra of NQ Lac and MY Per. The offset applied for plot clarity is given in brackets next to the star name. The asterisk means that a boxcar smoothing (with a window of 3 pixels) has been applied. The spectra are not corrected for reddening. Fluxes in units of $10^{-15} \mathrm{erg} \mathrm{cm}^{-2} \mathrm{~s}^{-1} \AA^{-1}$

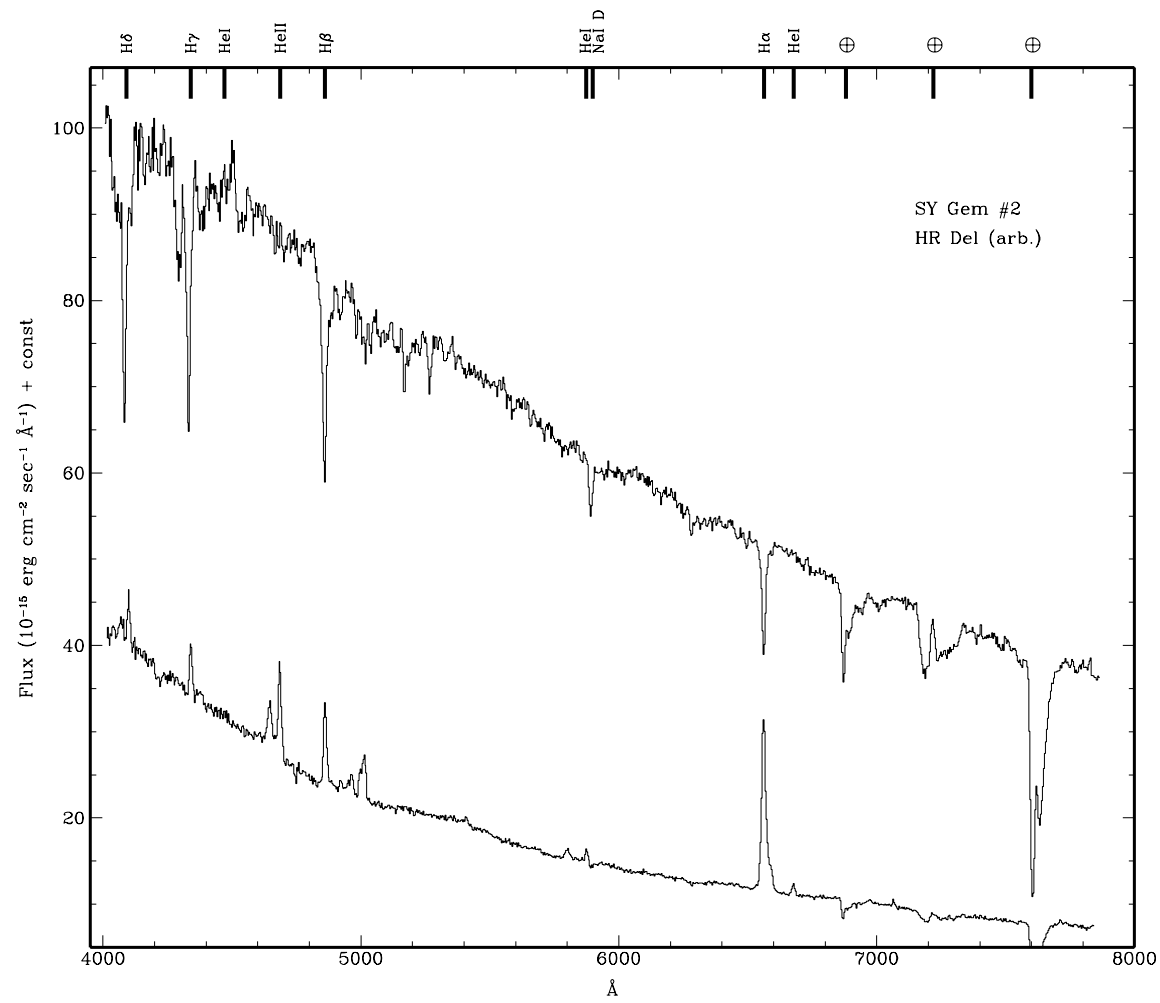

Fig. 6. Spectra of SY Gem \#2 and HR Del. The (arb.) notation means an arbitary zero point setting in the flux scale. The spectra are not corrected for reddening. Fluxes in units of $10^{-15} \mathrm{erg} \mathrm{cm}^{-2} \mathrm{~s}^{-1} \AA^{-1}$ 


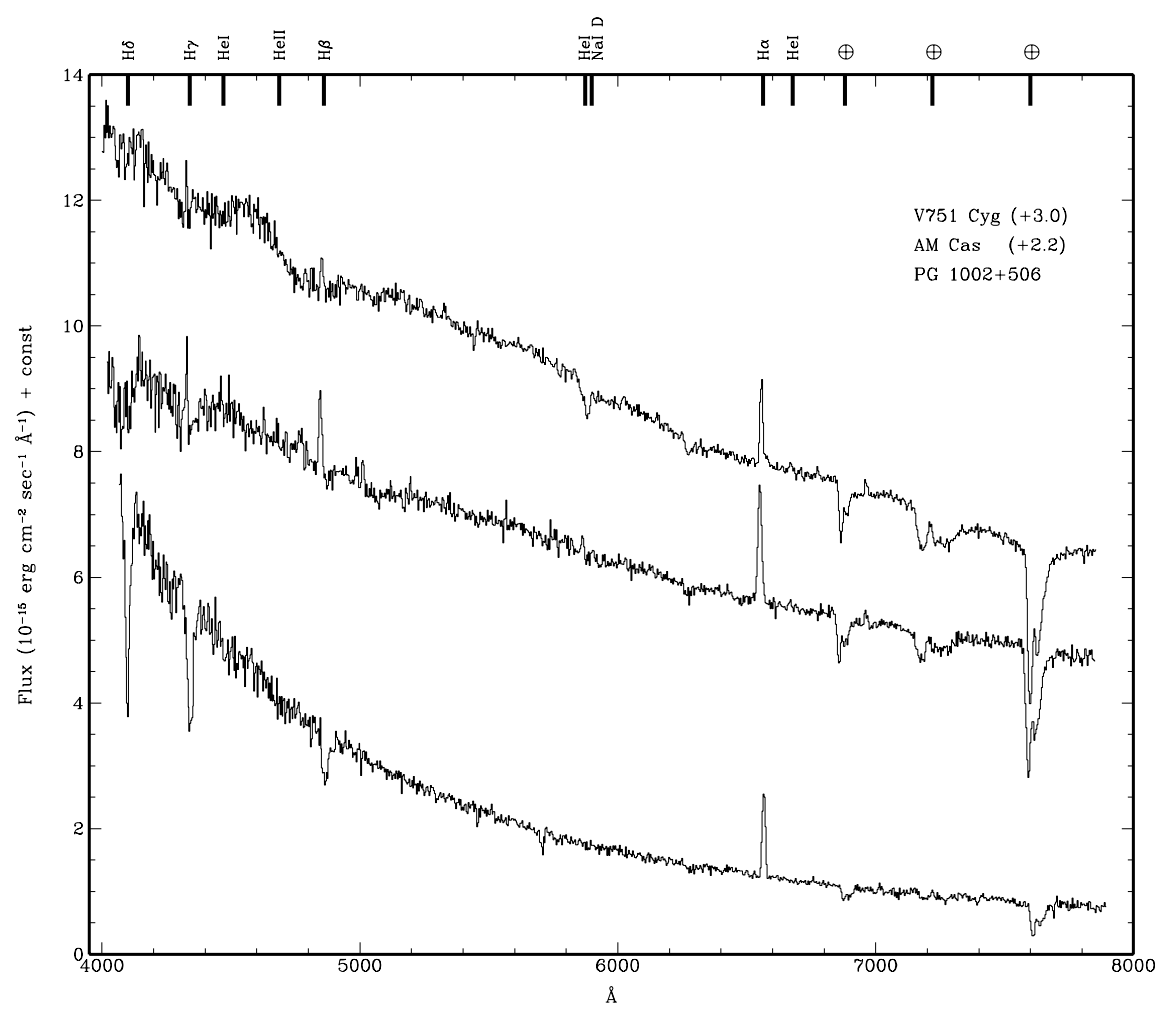

Fig. 7. Spectra of V751 Cyg, AM Cas (Loiano) and PG 1002+506. The offset applied for plot clarity is given in brackets next to the star name. The spectra are not corrected for reddening. Fluxes in units of $10^{-15} \mathrm{erg} \mathrm{cm}^{-2} \mathrm{~s}^{-1} \AA^{-1}$

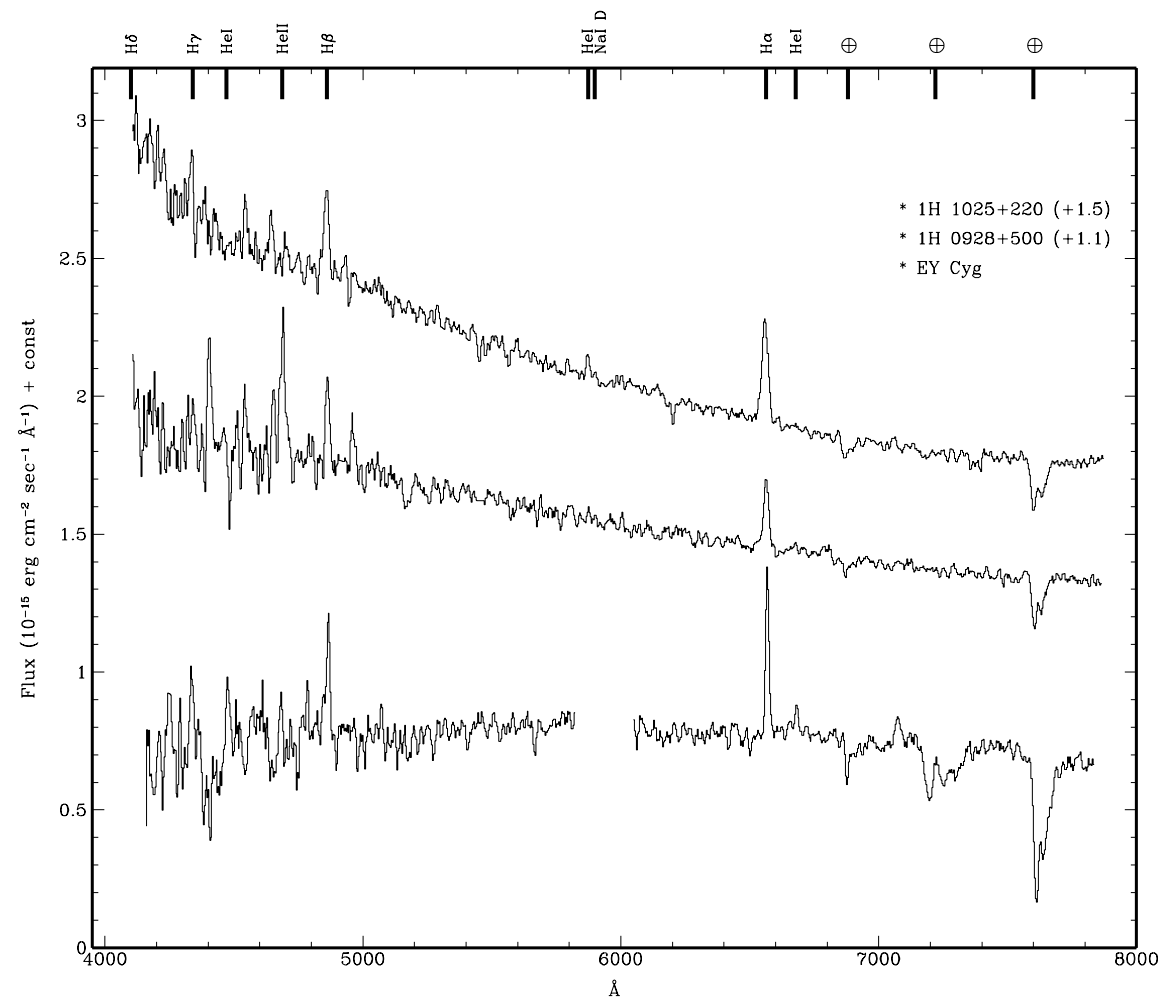

Fig. 8. Spectra of $1 \mathrm{H} 1025+220$, $1 \mathrm{H} 0928+500$ and EY Cyg. The offset applied for plot clarity is given in brackets next to the star name. The asterisk means that a boxcar smoothing (with a window of 3 pixels) has been applied. The spectra are not corrected for reddening. Fluxes in units of $10^{-15} \mathrm{erg} \mathrm{cm}^{-2} \mathrm{~s}^{-1} \AA^{-1}$ 


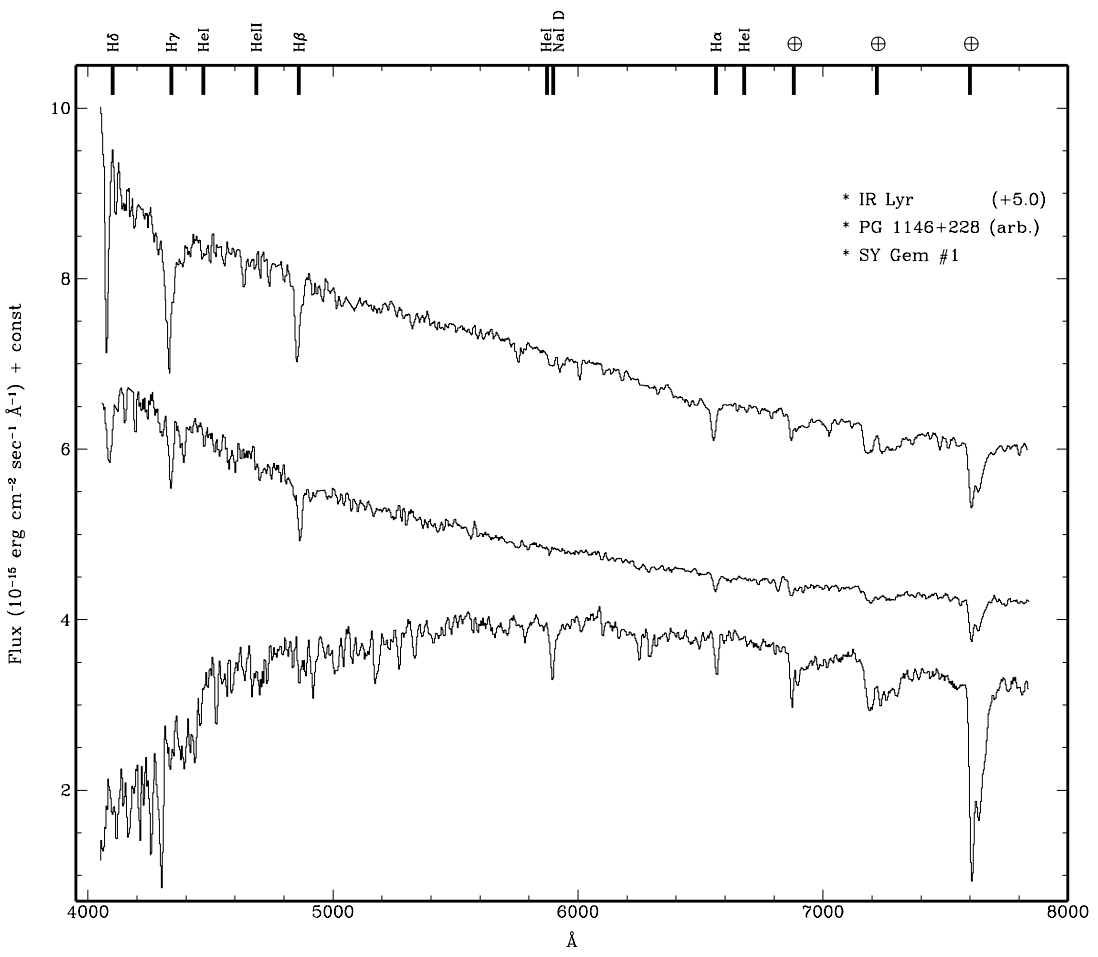

Fig. 9. Spectra of IR Lyr, PG $1146+228$ and SY Gem \#1. The offset applied for plot clarity is given in brackets next to the star name. The (arb.) notation means an arbitary zero point setting in the flux scale. The asterisk means that a boxcar smoothing (with a window of 3 pixels) has been applied. The spectra are not corrected for reddening. Fluxes in units of

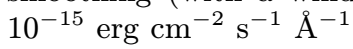

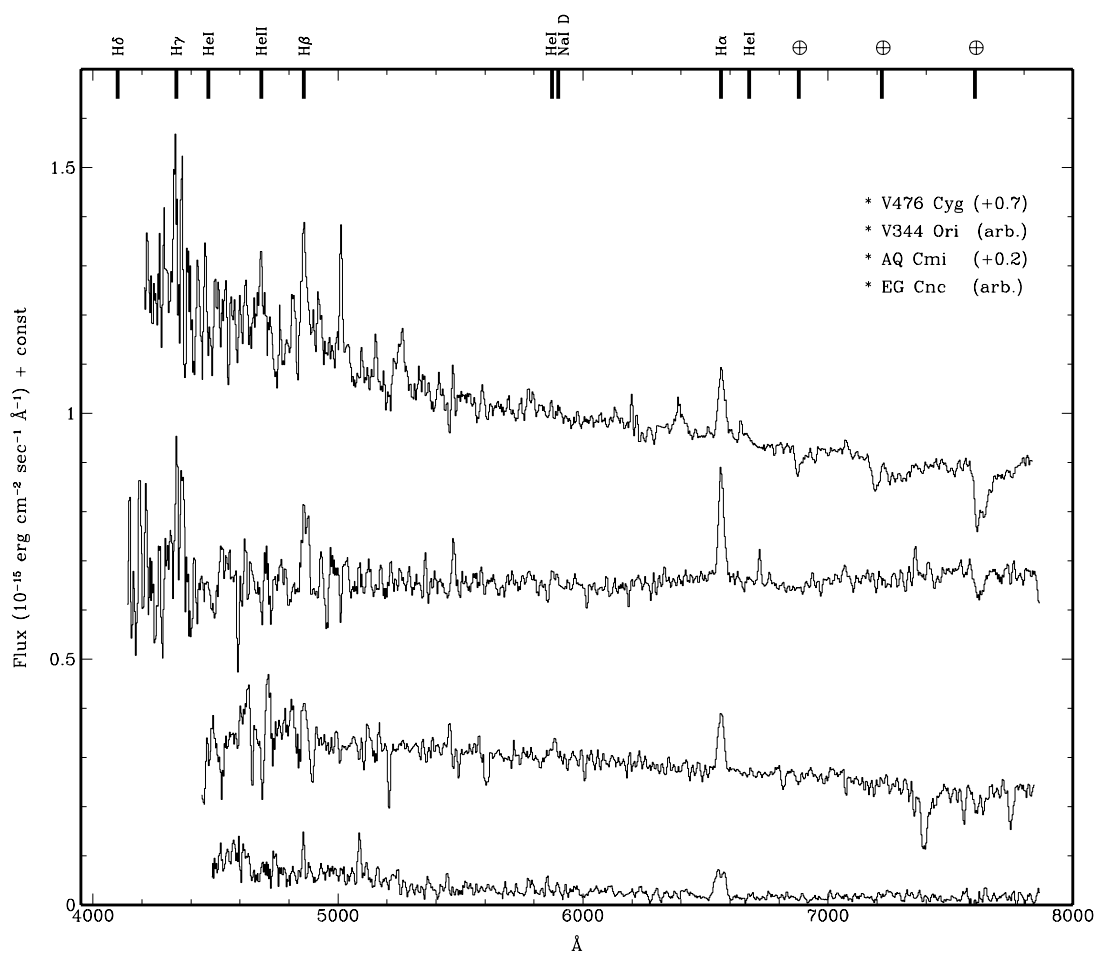

Fig. 10. Spectra of V476 Cyg, V344 Ori, AQ Cmi and EG Cnc. The offset applied for plot clarity is given in brackets next to the star name. The (arb.) notation means an arbitary zero point setting in the flux scale. The asterisk means that a boxcar smoothing (with a window of 3 pixels) has been applied. The spectra are not corrected for reddening. Fluxes in units of $10^{-15} \mathrm{erg} \mathrm{cm}^{-2} \mathrm{~s}^{-1} \AA^{-1}$ 